\title{
SEARCHES FOR BURSTS OF TEV GAMMA RAYS \\ ON TIME-SCALES OF SECONDS
}

\author{
V.Connaughton,M.Chantell,A.C.Rovero, T.Whitaker,T.C.Weekes \\ Whipple Observatory, S.A.O., P.O.Box 97, Amado, AZ 85645 \\ C.W.Akerlof,D.I.Meyer,M.S.Schubnell \\ University of Michigan, U.S.A \\ D.J.Fegan,S.Fennell,J.Hagan,N.A.Porter,M.Punch \\ University College, Dublin, Ireland \\ J.Gaidos,G.Sembroski,C.Wilson \\ Purdue University, U.S.A \\ A.M.Hillas, J.Rose,M.West \\ University of Leeds, U.K \\ A.D.Kerrick,P.Kwok,D.A.Lewis,R.C.Lamb,G.Mohanty \\ Iowa State University, U.S.A
}

\begin{abstract}
The Whipple Observatory gamma-ray telescope has a high sensitivity to sources of gamma rays in the 0.4 to $4 \mathrm{TeV}$ energy range. Although this sensitivity is used primarily to search for discrete sources of gamma-rays the instrument also has sensitivity to gamma-ray bursts on time-scales from milliseconds to seconds. The field of view is limited but the source location capability is good. Such bursts could radiate with peak luminosity at $\mathrm{TeV}$ energies and could originate from (a) primordial black holes or (b) cosmic strings; they could also be the high energy counterparts of BATSE-type bursts and hence of unknown origin. The search of the Whipple data-base for statistically unlikely consecutive events on time-scales of seconds will be described and compared with the theoretical predictions.
\end{abstract}

\section{INSTRUMENT}

The $10 \mathrm{~m}$ Optical Reflector at the Whipple Observatory on Mount Hopkins in southern Arizona (elevation $2.3 \mathrm{~km}$ ) has been used in studies of very high energy gamma rays and cosmic rays since 1968. It consists of 248 hexagonal mirrors mounted on a steel frame whose radius of curvature is equal to the focal length of the ground-glass, front- aluminized, mirrors. The point spread function has an angular width (FWHM) of less than $0.13^{\circ}$. The alt-azimuth mount can be programmed to track sidereally with an accuracy of $<0.1^{\circ}$. Since April, 1988, the reflector has been equipped with a fast High Resolution Camera consisting of 109 prnt's. The diameter of the inner 91 pixels is $0.25^{\circ}$ and the diameter of the overall field of view is $3.5^{\circ}$. The camera is triggered when two tubes exceed a preset threshold; the trigger rate is between 3 and $10 \mathrm{~Hz}$. 


\section{IMAGING TECHNIQUE.}

Gamma-ray showers are identified by shape and orientation of the recorded Cherenkov light image; more than $99.7 \%$ of the background can be thus rejected with loss of less than $50 \%$ of the signal.

The telescope is routinely used on all clear dark nights to search for discrete sources of very high energy gamma rays. To date two sources have been detected with some certainty: the Crab Nebula (Vacanti et al. 1991) and Markarian 421 (Punch et al. 1992). Because of the high statistical significance of these detections the imaging technique can be used in a search for gamma-ray burst phenomena.

The unique advantage of this instrument is that its response to gamma-ray showers is well-known and hence the signature of a gamma-ray burst can be identified with confidence. At the same time the recorded background events can be used as an effective check against the occurrence of false bursts i.e. nonstatistical clustering of triggers in time. This is particularly important for atmospheric Cherenkov detectors which are exposed to the night-sky and possible spurious sources of pulsed radiation.

Since 1977 the Whipple telescopes have been used in a variety of configurations to search for the gamma-ray emission from the final stages of evaporation of primordial black holes (Porter and Weekes, 1979). Most recently the High Resolution Camera on the $10 \mathrm{~m}$ optical reflector has been used in these searches (Nolan et al. 1990; Connaughton et al. 1991).

For the purposes of this search we define a candidate gamma-ray burst as an occurrence of $n$ events with the shape expected of a gamma-ray shower in a time $t$. In addition the axes of the gamma-ray candidate images should intersect; the point of intersection can be outside the geometrical field of view.

\section{OBSERVATIONS.}

A pilot search for bursts of $\mathrm{TeV}$ gamma rays was undertaken using data from the 1991-1992 season of data- taking with the 10m High Resolution Imaging Camera.

A total of 45 hours taken between April and June 1992 covering a variety of sources comprised the final database. The data files were parameterized and a reduced data set containing gamma-ray like events was defined by selecting events on the basis of the shape of its resulting image. No event selection was attempted using the orientation parameter of the image since the potential burst sources could lie anywhere within the field of view. For the same reason, the traditional method of comparing the 'ON' source region with a run taken in a suitable 'OFF' source region is not a valid way of defining the background data set in this search.

The background files were produced by taking each complete parameterized data file, scrambling all the events while maintaining the original time sequence, and applying the image cuts to the new scrambled file. The events surviving the cuts would be identical to those in the real reduced data set but they would have a random distribution in time. This scrambling process was repeated so 
that each file had 25 corresponding background files.

\section{METHOD.}

To search for bursts in the above data set, a 1s moving window was applied to each file. The window was positioned on each event in the file and its time was compared with the times of the previous events. In this manner, bursts of photon-like events were isolated and the occurrences of two to five events per second counted.

The numbers obtained were compared to random expectation by applying the same technique to each set of 25 scrambled files and calculating the average occurrence of 2 to 5 -fold coincidences.

A routine was developed to display the bursts as they appear on the PMT array of the detector by superimposing each reconstructed image on a $3^{\circ} \times 3^{\circ}$ grid along with the other images comprising the burst and their major axes.

\section{RESULTS.}

The observed number of bursts ( 3 candidate gamma-ray events in $1 \mathrm{sec}$ ) was 7 ; the predicted rate was 8.51 . The number can be further limited by consideration of the orientation of three images which must intersect; the tolerance for intersection is the cone defined by the angle, alpha $<15^{\circ}$. In addition the displacement from the centroid of the image to the point of origin is a function of the ellipticity of the image. Only one of the seven candidate bursts satisfies these criteria; hence we derive an upper limit (at the $99 \%$ level) of 6.1 events in $1.64 \times 10^{5} \mathrm{sec}$ or $1116 \mathrm{yr}^{-1}$.

\section{THE PRIMORDIAL BLACK HOLES PHENOMENON.}

In the early Universe primordial black holes (PBH) of arbitrarily small masses may have formed; the simplest mechanism is the gravitational collapse of overdense regions. Such PBH's in the Universe would go largely unnoticed were it not for the Hawking radiation; in particular the final stages of black hole evaporation may produce a burst of very high energy gamma-ray radiation that can be detected in ground-based telescopes.

\section{A NEW LIMIT ON THE PBH DENSITY.}

The gamma-ray burst limit derived above can be used to set a limit to the PBH density. The PBH burst luminosity used is that given in eqn. (16) in Halzen et al. (1991); for dt $=1 \mathrm{sec}$ and $E_{D}=0.5 \mathrm{TeV}$ we get $N_{\text {gamma }}=8 \times 10^{28}$. We can thus determine the maximum distance that a $\mathrm{PBH}$ could be from the detector and still be detected; this is equal to $0.5 \mathrm{pc}$. For a collection area of $1 \times 10^{9} \mathrm{~cm}^{2}$ and solid angle of $3 \times 10^{-3}$ ster, we find the sensitive volume, $\mathrm{V}=1.2 \times 10^{-4} \mathrm{pc}^{3}$. The upper limit to the $\mathrm{PBH}$ density,

$$
N_{\mathrm{pbh}}=R / V=1116 / 1.2 \times 10^{-4}=9.3 \times 10^{6} \mathrm{PBH}^{\prime} \mathrm{s} \text { per } \mathrm{pc}^{3} \text { per } \mathrm{yr} .
$$

It should be noted that the limit from this pilot search is based on the analysis of less than $10 \%$ of the data-base from just one of the five years of 
observations in the Whipple Observatory High Resolution Camera data-base A more significant limit based on observations at a much higher energy threshold ( $>50 \mathrm{TeV}$ ) over a 3.3 year observing period has recently been published (Alexandreas et al. 1993).

\section{PREVIOUS PBH LIMITS REVISITED.}

The original prediction of the expected burst power was that there would be $10^{30}$ gamma rays of energy $5 \mathrm{TeV}$ emitted in $0.1 \mathrm{sec}$. No significant evidence was found for bursts with these characteristics and upper limits were derived based on this prediction. A re-evaluation of the final stages of $\mathrm{pbh}$ evaporation (Halzen et al. 1991) reduces the predicted emission and broadens both the spectrum and duration of the expected gamma-ray bursts. It is therefore necessary to revise the previously derived upper limits; these are listed below.

\begin{tabular}{l|l|l|l}
\hline \hline Experiment & Reference & $N_{\mathrm{pbb}}$ & $\begin{array}{l}\text { New limit } \\
\mathrm{pc}^{-3} \mathrm{yr}^{-1}\end{array}$ \\
\hline $10 \mathrm{~m}$ & Porter+Weekes, 1979 & $7.1 \times 10^{28}$ & $6.0 \times 10^{8}$ \\
S.L.'s & " & $7.7 \times 10^{28}$ & $4.9 \times 10^{7}$ \\
$10 \mathrm{~m}$ (A) & Nolan et al,1990 & $3.0 \times 10^{28}$ & $9.3 \times 10^{6}$ \\
$10 \mathrm{~m}$ (B) & " & $3.0 \times 10^{28}$ & $1.8 \times 10^{6}$ \\
$10 \mathrm{~m}$, high z & Connaughton et al, 1991 & $2.6 \times 10^{27}$ & $8.7 \times 10^{7}$ \\
\hline
\end{tabular}

More recent work (Cline et al. 1993) suggests that uncertainties in the particle physics of the density of states of the final stages of $\mathrm{PBH}$ evaporation mean that a search based on a 1 s time-scale is too restrictive and that future searches should involve windows ranging from $10^{-7} \mathrm{~s}$ to a few seconds.

\section{GAMMA-RAY BURSTS FROM SUPERCONDUCTING COSMIC STRINGS.}

The detection of a burst of $\mathrm{TeV}$ photons or neutrinos offers one of the few possibilities of directly detecting a cosmic string. Because of the greater sensitivity of $\mathrm{TeV}$ gamma-ray telescopes the photon detection appears more likely. Two possibilities are considered:

(a) Cosmic Strings

Ordinary (non-superconducting) Cosmic Strings (CS's) release extremely energetic particles in the cusp region on very short times-scales; these particles decay down to gamma rays and neutrinos which are emitted in narrow beams. MacGibbon and Brandenberg (1993) have calculated the maximum photon cusp radiation from individual CS's and compared it with the detection capabilities of existing ground-based gamma-ray telescopes. The duration of the signal at the detector is determined by the spreading out of the photon arrival times due to the decay process; this time is difficult to predict.

(b) Superconducting Cosmic Strings

Some kinds of cosmic strings behave like superconductors. In a recent study 


\section{Searches for Bursts of TeV Gamma Rays}

(Samura and Kobayakawa, 1993), the gamma-ray emission from Superconducting Cosmic Strings (SCS's) was evaluated; the SCS emits heavy fermions in a jet when it reaches a saturated current. If the mass of fermions is greater than a few hundred TeV, the resultant burst from a SCS out to a distance of $1 \mathrm{kpc}$ was shown to be detectable to a telescope with the sensitivity of the Whipple $\mathrm{HRC}$; the time-constant of the bursts was 1 sec.

For a telescope with the sensitivity of the Whipple telescope they show that the absence of any detected bursts in a year of operation can set an upper limit to the number of strings of $10^{8}$ out to a distance of $10 \mathrm{kpc}$.

\section{BATSE COUNTERPART SEARCH.}

To extend the spectra of GRB's to higher energies it is desirable to have simultaneous coverage of a burst by BATSE and an atmospheric Cherenkov telescope. Because of the limited field of view and low duty-cycle of the latter, this is not easy to achieve.

As shown above the Whipple atmospheric Cherenkov telescope has an energy threshold of about $0.5 \mathrm{TeV}$ and a burst sensitivity of about 3 photons for a burst of duration $1 \mathrm{sec}$. A burst of 3 events corresponds to about $2.4 \mathrm{ergs}$, a fluence of

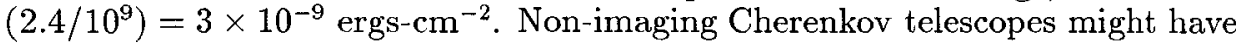
a factor of 2 lower sensitivity. This is better than BATSE and suggests that all the BATSE bursts with flat spectra (differential power index $=-2$ ) would be detectable by existing Cherenkov telescopes if their fields of view overlapped.

The probability of overlap in a single year with a single atmospheric Cherenkov telescope is small; however given the anticipated length of the CGRO BATSE mission ( $>8$ years) and the number of atmospheric Cherenkov telescopes now in operation $(\sim 10)$, the probability of overlap is $>1$. We have begun a search for the overlap with ground-based telescopes in the first few years of BATSE operation. For this search we are using the observational data-bases of the following atmospheric Cherenkov observatories: Whipple Observatory, U.S.A. (Mt. Hopkins); CANGAROO, Adelaide-Tokyo (Woomera, Australia); Crimean Astrophysical Observatory, Ukraine; Themistocle, France (Themis); Institute of High Energy Physics, Beijing: Tata Institute, India (Pachmari); Potchefstroom, South Africa; Durham, U.K. (Narrabri, Australia); Wisconsin-Purdue (South Pole).

\section{REFERENCES}

Alexandreas, D.E. et al., 1993, Proc. 23rd ICRC (Calgary), 1, 428. Chantell, M. et al. 1992; Proc. CGRO Symposium (St. Louis), 833. Cline, D., Hong, W., 1993, Proc. of this workshop. Connaughton, V., et al. 1991; Proc. 22nd ICRC (Dublin), 1, 69. Connaughton, V. et al., 1993, Proc. 23rd ICRC (Calgary), 1, 112. Halzen, F. et al. 1991; Nature, 353, 807. MacGibbon, J.H., Brandenberger, R.H., 1993: Phys. Rev. D. 47, 2283. Nolan, K. et al., 1990, Proc. 21st ICRC (Adelaide), 2, 150. Samura,T. and Kobayakawa, K., 1993, Proc. 23rd ICRC (Calgary), 1, 128. Porter, N.A. and Weekes, T.C., 1979, Nature, 277, 199. Punch, M. et al., 1992, Nature, 358, 477. Reynolds, P.T. et al., 1993, Ap. J. 404, 206. Vacanti, G. et al., 1991, Ap.J. 377, 467. 